\title{
Burke, Andree / Hiepel, Ludger / Niggemeier, Volker / Zimmermann, Barbara (Hg.) (202I). Theologiestudium im digitalen Zeitalter. Stuttgart: Kohlhammer. ISBN 978-3-1 7-038893-2. 364 Seiten.
}

\author{
Andreas Menne \\ Johannes-Gutenberg-Universität Mainz (andreas.menne@uni-mainz.de)
}

Die Digitalisierung stellt einen der wohl umfassendsten technischen und sozialen Transformationsprozesse unserer Zeit dar. Der damit verbundene gesellschaftliche Wandel ist so weitreichend, dass im Hinblick auf unser alltägliches Handeln schon länger mit guten Gründen von einer ,Kultur der Digitalität' gesprochen werden kann. Nicht zuletzt im Rahmen der Corona-Pandemie tritt deutlich zu Tage, wie sehr digitale Praktiken zum Standardrepertoire vieler Menschen des 21. Jahrhunderts gehören. Sie greifen wie selbstverständlich auf diese zurück, um ihr Leben in den unterschiedlichsten Kontexten zu bestreiten und die damit verbundenen Herausforderungen zu bewältigen. Insofern erscheint es mehr als angebracht, auch Bildungsprozesse wie das Theologiestudium vor dem Hintergrund solcher Praktiken und Prozesse zu reflektieren. Diesen Versuch unternehmen die Herausgeber*innen und Autor*innen des vorliegenden Sammelbandes. Ziel des Bandes ist es, ,alle, die mit dem Fach ,Katholische Theologie' verbunden sind, darin zu bestärken, digitalen Transformationen mit offenem Visier zu begegnen" und zu eruieren, „welches [digitale, AM] Rüstzeug Absolvent*innen eines theologischen Studiums für unterschiedliche Berufsfelder mitbringen" (Burke et al., 9).

Hervorgegangen ist die Veröffentlichung aus den zweiten Münsteraner Fachgesprächen zur Zukunft des Theologiestudiums im Frühjahr 2019. Die darin abgedruckten Artikel beruhen demnach auf Reflexionen, die bereits vor Beginn der Pandemie angestoßen wurden. An vielen Stellen fließen jedoch die intensiven Erfahrungen mit der digitalen Lehre des vergangenen Jahres in die Texte ein. Dies bereichert den Band einerseits um wichtige Erkenntnisse aus der Praxis. So werden etwa die Ambivalenzen einer digitalisierten, aber aus didaktischer Sicht deshalb noch nicht zwingend innovativen Lehre sichtbar gemacht und kritisch geprüft. Andererseits steht an einigen Stellen die Frage im Raum, welche Gültigkeit diese Erfahrungen für eine digitale Lehre ,nach dem Ausnahmezustand' besitzen.

Die Publikation versammelt 26 Beiträge von 35 Autor*innen. Der Kreis der Verfasser*innen ist entsprechend der Anlage als Fachgespräch auf das Theologiestudium zugespitzt, aber unter dieser Prämisse bunt zusammengesetzt. So tragen neben Studierenden und Lehrenden der Theologie auch Mitarbeiter*innen aus der Studienverwaltung und dem Bibliothekswesen, Lehrkräfte und kirchlich Angestellte sowie eine gute Handvoll Forschender von anderen Studienstandorten ihre Perspektiven bei. Seine weitgehend überzeugende Struktur erhält der Sammelband bei aller personellen und thematischen Breite durch die Anordnung der Beiträge entlang des aus der elektronischen Datenverarbeitung entlehnten EVA-Prinzips: Eingabe, Verarbeitung, Ausgabe. In der Eingabe werden ausgewählte digitale Transformationsprozesse aus der Lebenswelt der am Theologiestudium beteiligten Personen in den Blick genommen. Die Verarbeitung beansprucht diese Einblicke „zu systematisieren und aktuelle Bewältigungsversuche des digitalen Wandels zu reflektieren" (10), bevor in der Ausgabe deren Konsequenzen für die Hochschullehre von Vertreter*innen unterschiedlicher theologischer Fächer bedacht werden.

Die digitalen Phänomene, die so in den Blick genommen werden, sind vielfältig. Sie reichen von Beobachtungen religiöser Akteur*innen in den sozialen Medien (Klaholz \& Schmidt, 25-30; Wode, 31-35) und der Rolle von Religion in Computerspielen (Zirpel, 37-43), führen von dort über die Präsentation von digitalen Tools für die kompetenzorientierten Hochschullehre (Donath et al., 165-185) sowie die 
Reflexion ihrer ambivalenten didaktischen Auswirkungen (Reis, 67-85; Hunze, 97-119) und gehen bis hin zu der Frage nach spezifischen Kompetenzen im Umgang mit digitalen Technologien theologischer Forschung wie XML-Datenbanken (Hiepel \& Niggemeier, 208-209; Brockmann et al., 223-226) oder virtueller Kartographie (Brockmann et al., 226-227). Aber auch ethische Implikationen des digitalen Wandels werden bedacht (Hein, 265-272). Nur vereinzelt zieht man dabei Konzepte des interdisziplinären Diskurses um digitale Bildung heran und befragt diese auf ihre Tragfähigkeit für die Theologie. Neben inzwischen etablierten (hochschul-)didaktischen Schlagwörtern wie Blended Learning oder der Unterscheidung zwischen synchronen und asynchronen Lernsettings (Zimmermann, 64-65) finden auch umfassendere Zugänge wie das SAMR-Modell (Reis, 74-75; Mansfeld \& Schoch, 346-350), die KMK-Strategie (Hunze, 102-103) oder der Computational Thinking-Ansatz (Harrich \& Hiepel, 87-96) Berücksichtigung. Die Beiträge veranschaulichen damit in ihrer Summe zwar, wie breit das Feld digitaler Kompetenzen im Theologiestudium bestellt sein könnte; zugleich wird aber auch deutlich, dass diese lediglich in Ansätzen erschlossen werden und auch der Zugriff auf etablierte Modelle digitaler Bildung exemplarisch bleibt.

Besonders gewinnbringend spannt sich der konzeptionelle Bogen zwischen Eingabe und Ausgabe auf. Formulieren die studentischen Autor*innen zu Beginn, sie erhofften sich zum einen „eine Digitalisierung von Inhalten und Didaktik in der universitären Lehre“ und zum anderen „das Digitale als selbstverständliche Dimension des Menschseins zu verstehen und gerade in der Theologie zu reflektieren“ (Bartnick et al., 17), antworten die hinteren Beiträge des Bandes ganz konkret auf diesen Wunsch. Einige Fachvertreter*innen beschreiben detailliert, welche digitalen Praktiken und Technologien vermehrt Eingang in die Forschung ihres Faches gefunden haben (Hiepel \& Niggemeier, 205-206; Brockmann et al., 218-227; Hanke \& Puzio, 251-254) und entwickeln erste Formulierungen für digitale Kompetenzanforderungen und -raster der theologischen Lehre (Hiepel \& Niggemeier, 207-210; Hanke \& Puzio, 263; Hein, 272-274; Mansfeld \& Schoch, 352-358). Andere Artikel vollziehen Reflexionsbewegungen auf das Leben in einer digitalen Gesellschaft (Becker, 237-244; Heidkamp, 334-337) oder beschreiben, wie sich theologische Forschungsfragen, -haltungen und Fachinhalte im Zuge der Digitalisierung verändern (Weißer, 284-287; Burke \& Harrrich, 294-300; Lüstraeten, 304-310; Neumann, 320-327). Die Verarbeitung hingegen entfernt sich zuweilen von diesem auf das Theologiestudium fokussierten Profil des Bandes und präsentiert abstraktere Gedanken zum digitalen Wandel (Kirchschläger, 121-137; Henkel, 187198). Nichtsdestotrotz entwickeln einige der Beiträge in diesem Teil der Veröffentlichung mit Schlagworten wie dem „Lernen über, durch und mit Digitalisierung“ (Hunze, 111) oder der These einer digitalen „Implosion des hochschuldidaktischen Dreiecks“ (Reis, 78) hilfreiche begriffliche Werkzeuge zur Analyse digitaler Bildung im Fach Theologie. Auch der vermeintliche Spezialdiskurs um ein theologisches Fernstudium erweist sich als lehrreich, wenn etwa die vorgestellten Evaluationen zweier Studiengänge verdeutlichen, dass digitale und präsentische Lehre keinesfalls als reine Gegensätze verstanden werden sollten (Adams \& Weirer, 139-152; Feeser-Lichterfeld, 153-163).

Der Sammelband führt damit beeindruckend vor Augen, wie eine theologische Fakultät ihren hochschuldidaktischen Reflexionsprozess über digitale Bildung anstößt. Die Beiträge markieren erste Potentiale einer theologischen Forschung und Lehre, die sich ihrer Digitalität bewusst wird. Dies demonstrieren insbesondere die stellenweise ausformulierten digitalen Kompetenzen für das Theologiestudium. Sie verleihen der theologischen Befassung mit dem Thema bei aller Exemplarität eine gewisse Systematik und können damit auch eine Inspiration für andere Studienstandorte sein. Zugleich verdeutlichen die Beiträge des Sammelbandes, dass die Theologie bezüglich des Nachdenkens über ihre digitalen Bildungsanliegen noch am Anfang steht. Neben spezifisch theologischen Auseinandersetzungen bedarf es, will man den Ansprüchen eines Studiums „im digitalen Zeitalter“ tatsächlich gerecht werden, in Zukunft auch eines fächerübergreifenden Austauschs mit Disziplinen wie den Medien- und Kommunikationswissenschaften, der Informatik oder den Fachdidaktiken und Bildungswissenschaften. Die ersten Schritte, die der Sammelband in diese Richtung geht, reihen sich in den Auftakt einer größeren Bewegung um eine Bildung in der digitalen Welt ein. 